\title{
What Became of the Genetic Challenge to Adam and Eve?
}

\author{
William Lane Craig \\ https://doi.org/10.54739/e7r9
}

oes population genetics challenge a single-couple origin to humanity? In early 2017, Dr. Richard Buggs raised scientific concerns about Dennis Venema's book, Adam and the Genome. His concerns were distinct and addition to concerns that Dr. Swamidass raised that year too. In late 2017, Dr. Venema responded at BioLogos, and a conversation grew. Together, several scientists did indeed find several fundamental mistakes with Dr. Venema's argument against a single couple origin of humanity. Eventually, in 2020, even BioLogos began backing away from Venema's scientific claims. In this article, Craig summarizes for a lay audience the science behind this shift. For technical readers, the forum thread for this article expounds and clarifies his summary with greater precision.

The traditional view of Adam and Eve as the fountainhead of all humanity has been vigorously challenged by some on the basis of the science of population genetics.

In order to understand this challenge, it is important to understand that according to the theory of evolution, and perhaps contrary to popular impression, evolution does not proceed along an isolated individual line. It is not as though some sequence of individual reptiles evolved, for example, into the first bird. Rather, the idea is that whole populations evolve over time. So the ongoing front of evolution is not like a pointed spear; it is rather like a broad front, as a whole population of organisms evolves together over time. In this case, a whole population of reptiles would be evolving bird-like characteristics. Similarly, in the case of human evolution, there is a whole population of hominins - man-like primates- which is gradually evolving characteristics of modern human beings. So, it is typically thought that there weren't originally two human persons - a couple - that were the ancestors of everybody else. Rather, modern human beings evolved through a whole population of hominins moving gradually toward more recognizable modern human forms.

But why couldn't there have been within this wider population of nonhuman hominins a first couple who made the transition to humanity and whose descendants became the human race? Some critics have alleged that this scenario is impossible, since it contradicts the data of population genetics. In order to understand this challenge, let's briefly review what we learned in high school biology.

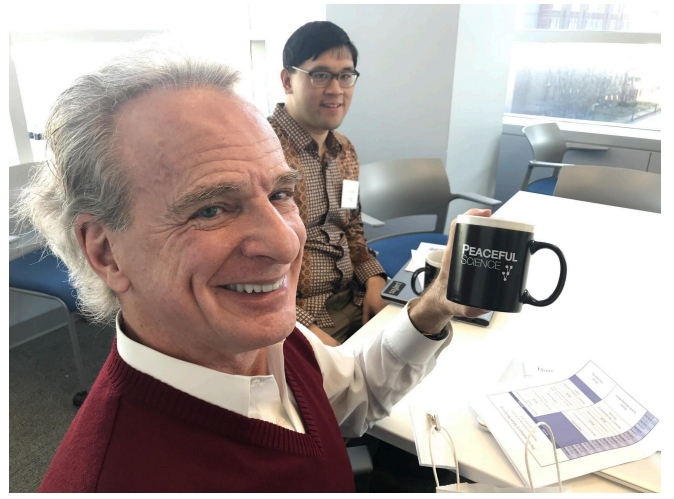

This article is a footnoted transcript of Dr. Craig's Defenders class earlier this month.
Dr. Craig holds that Adam and Eve were ancient, but The Genealogical Adam and Eve explores a recent Adam and Eve. See the discussion between Dr. Swamidass and Dr. Craig here about this difference. As Richard Buggs puts it, "Is the most important thing about them the time in which they existed, or something that made them objectively unique?"

Human beings have in each nucleus of each cell of their bodies 23 pairs of chromosomes containing the DNA that determines our genes. A segment of DNA is called a locus (Latin for "place"). The sequence of DNA letters at any locus is called an allele. Since our chromosomes come in pairs, we therefore have one allele at a locus on one chromosome and another allele at a similar locus on the other chromosome. These alleles determine features like eye color, height, skin color, and so on. Now the claim is that when we look at the genetic profile of the human population today, it is impossible that it could have stemmed from an original couple alone; there had to be numerous ancestors from the very beginning in order for the human race to have arrived at its present condition.

\section{The Problem}

So what's the problem supposed to be? Computational biologist Joshua Swamidass distinguishes at least four different problems that have been put forward in the popular literature as incompatible with an original human couple:

1. Multiplicity of alleles: There are just too many different alleles in the present population to have all come from an original human couple within the last 18 million years, which is long before human beings ever appeared on this planet.

2. Effective population size estimates: Various independent methods of estimating past population size all concur that the human population in the past was never fewer than around 10,000 people.

3. Trans-species variation: In order for all the alleles which we have in common with chimpanzees to be passed on to us from a common ancestor, there needed to be more than one couple who transmitted these genetic lines from that ancestor to us. In other words, a single couple could not have passed on to us all the genetic material which we share with chimps.

4. Divergence of alleles: To grasp this point it is vital to differentiate genetic divergence from genetic diversity. I shall take "genetic diversity" or "variation" to refer to the multiplicity of alleles in a 
population. ${ }^{1}$ Genetic divergence, on the other hand, has to do with the mutational distances between alleles in a population. We can visualize divergence by representing alleles as dots plotted on a plane. The more mutations separating two alleles, the greater the distance between them on the plot. One way to measure divergence would be to measure the distances of the farthest alleles from the most central allele. Draw a circle around all the dots, and the radius of that circle provides a measure of their genetic divergence.

Notice that the multiplicity of alleles (what I'm calling genetic diversity) is irrelevant; what matters is the spread of the alleles (genetic divergence). The wider the spread, the more ancient the most recent common ancestor of those alleles. We want to compute genetic divergence across the whole human genome. We can then ask how long and how rapidly mutations must have been occurring for the distances separating alleles in the present population to arise from a pair of sole genetic progenitors. Given the known mutation rate, we can use genetic divergence to calculate the time back to the most recent common ancestor (TMRCA). The argument is that it would take millions and millions of years for the observed divergence of alleles to have arisen from a common ancestor. We cannot reach single pair of sole genetic progenitors of the human race within the time during which hominins have existed on the earth. As one writer put it, this would make Adam literally “a monkey's uncle!”2 Therefore, there could not have been an original human pair from whom we all descend.

On the basis of evidence such as the above biologist Dennis Venema expresses supreme confidence that humanity did not descend from a single human couple:

Some ideas in science are so well supported that it is highly unlikely new evidence will substantially modify them, and these are among them. The sun is at the center of our solar system, humans evolved, and we evolved as a population. ${ }^{3}$

He here expresses what has been called " heliocentric certainty" against an original human pair. ${ }^{4}$

\section{The Problem Analyzed}

Swamidass has subjected the above arguments to searching criticism.

\section{Multiplicity of Alleles and Population Size}

Swamidass dismisses arguments based upon (1) and (2), as "just wrongheaded. These arguments are total misdirections that have nothing to do with the key question. They are category errors." ${ }^{5}$ From the mere number or variety of alleles in the human population today, nothing at all follows about population sizes in the deep past. What matters is the divergence of alleles in the population. The argument from genetic diversity, as I have defined the term, is a red herring.

Arguments based on estimated effective population size are misleading because such estimates are averages over a window of time and so are consistent with peaks and valleys within the intervals. Venema consistently errs in taking these estimates to concern minimum population size rather than average population size. ${ }^{6}$ In
2017, geneticist Richard Buggs pointed out that the hypothesis of a bottleneck of two people had in fact never been tested scientifically. ${ }^{7}$

Indeed, as Swamidass observes, we know that at some point in the past the number of human beings goes to zero and therefore to fewer than 10,000 individuals. In thinking otherwise, Venema is guilty of a crucial equivocation between "ancestors" and "humans." Even if the ancestral population of hominins leading to humans remains constantly above several thousand, it does not follow that there were not at some time exactly two humans who emerged within that population. It is entirely possible that at some time in the past, the total number of humans was exactly two, even though the total population of hominins at the time was much greater. The descendants of these early humans might or might not have interbred with their non-human contemporaries. If they did, then the founding couple would not be our sole genetic progenitors, for outsiders would have had genetic input into the human race. On the other hand, if such interbreeding never occurred, the founding couple would be the sole genetic progenitors of the human race, there being no outside input. In either case, there could have been an original founding pair.

\section{Trans-Species Variation}

What, then, of the argument from trans-species variation? While initially plausible, the argument dissolves upon examination. Since every human being has two sets of similar chromosomes (not counting the $\mathrm{X}$ and $\mathrm{Y}$ chromosomes determining sex), a founding human pair can together carry at most four alleles at any locus into the descendant population. So if it could be shown that there are more than four allele lineages exhibiting trans-species variation, then we should have strong evidence against an originating human pair. But apart from an outdated study by Francisco Ayala, there is no evidence for this. Although this issue is still under debate, Swamidass reports that other studies have failed to uncover evidence of transspecies variation between humans and non-human ancestors involving more than four allele lineages. Even if such variation should be discovered, it could indicate no more than the fact that Adam and Eve were not our sole genetic progenitors, but that there was interbreeding with outsiders, who introduced more alleles into the human population.

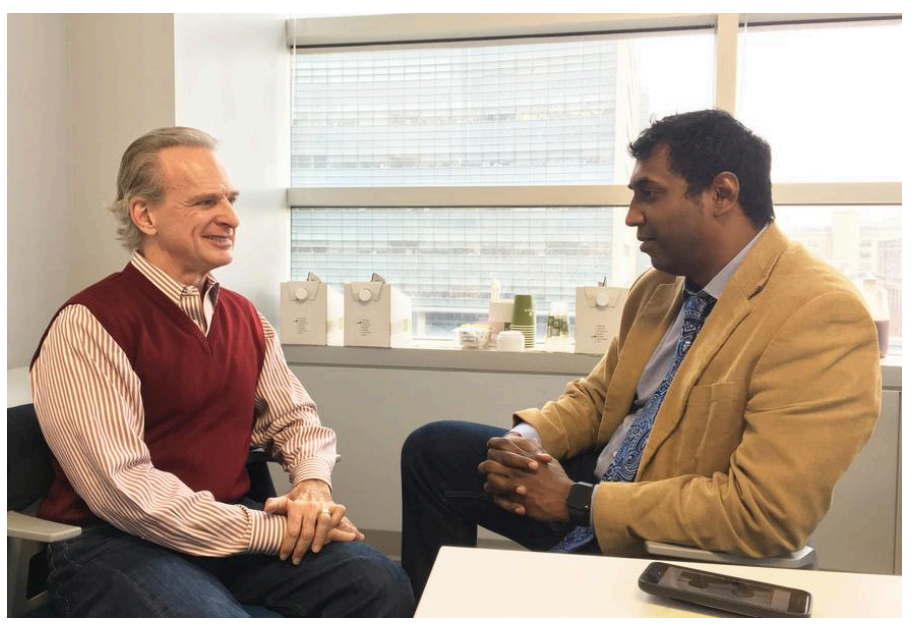

Dr. Craig attended the 2019 Peaceful Science workshop on <em>The Genealogical Adam and Eve $</$ em $>$, which explored a recent Adam and Eve. He, however, prefers an \&ldquo;ancient\&rdquo; genealogical Adam and Eve. 
Moreover, there is a plausible alternative explanation of trans-species variation among hominins, namely, convergent evolution. In convergent evolution, similar alleles evolve independently in different species. Convergent evolution seems to be common among hominin species, producing similarities, for example, bipedalism, among independent species that may be misleading in determining lines of descent. Swamidass notes that the particular gene studied by Ayala is "one of the genes with clear evidence of convergent evolution." 9 This finding robs the argument from trans-species variation of its force.

\section{Genetic Divergence}

The decisive question, then, concerns the divergence of alleles in the human population. Swamidass points out that a founding couple could have been heterozygous, each carrying two different alleles at any locus of their chromosome pairs, for a total of four alleles between the two of them for any locus to be passed on to their descendants. ${ }^{10}$ In that case the relevant time is not the TMRCA but the time to the most recent four alleles (TMR4A). Population genetics has been concerned only with the TMRCA, so that no studies of the TMR4A had been published prior to Swamidass' work, requiring him to do his own original modeling in order to obtain a date. I'll skip the fascinating details and cut to the chase. Swamidass determines a date of $495 \pm 100$ kya for the TMR4A. So there could have been a founding couple about 500 kya who were the sole genetic progenitors of mankind. On the basis of this work, Richard Buggs agreed that Swamidass, for the first time, had tested the hypothesis of an original human pair.

More recently these findings have been confirmed by Ola Hössjer and Ann Gauger, who explore what they call a Single Couple Origin (SCO) model of the human race. ${ }^{11}$ They find that the data are consistent with at least two different SCO models: (1) A model featuring a homozygous

first couple dating to about 2 mya and (2) a model featuring a heterozygous first couple who lived about 500 kya ago. Thus, they conclude, given common assumptions shared by evolutionary geneticists, a single-couple origin is possible, despite claims to the contrary.

So while a recent bottleneck is ruled out by the genetic divergence exhibited by today's human population, a bottleneck before 500 kya is possible, in which case the founding pair would be the common ancestors of Homo sapiens, Denisovans, and Neanderthals. "The dust has yet to settle on the scientific details," says Swamidass, "But it looks likely at this point that a bottleneck anytime before 700 kya is undetectable in genetic data." ${ }^{12}$ Such a date well within the range of our proposed classification of Adam as Heidelberg Man.

After extended discussion with Buggs, Swamidass, and others, Venema came to acknowledge in 2019 the failure of his

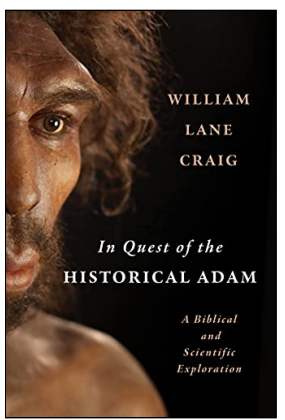

This article was refined and included within Craig's book, *In Quest of the Historical Adam*.

arguments against a single couple origin. "Based on some new simulations and some other published studies that we drew on, our group came to an agreement - that if an event like this had happened, we would be able to detect it if it happened more recently than 500,000 years ago. That was surprising to me, to be sure - I thought beforehand that an event like that would show up even further back in time." ${ }^{13}$

Venema nonetheless insists that, despite the possibility of a founding pair before $500 \mathrm{kya}$, the existence of such a couple is highly improbable. "In order for this to work, one would have to propose that in one generation all of them were obliterated, save two."14 This bold claim is obviously false, since a founding pair could have existed as part of a wider population with whom the founding pair's descendants may or may not have interbred.

It is important to understand that the existence of a historical Adam and Eve need not imply their sole genetic progenitorship, especially over tens of thousands of years. Even if their descendants were for a time reproductively isolated, such isolation could result, not from population reduction, but from social distancing due to a myriad of factors, including geographic isolation, tribalism, language barriers, xenophobia, cognitive capacity differences, racism, just plain revulsion, and so on, as well as any population reduction we might imagine. Perhaps these barriers were sometimes breached, but then we do not have any idea whether there were offspring of such unions that had genetic input into the human line. Of course, once Adam and Eve's descendants replaced Heidelberg Man, we know that there was interbreeding among the extended human family, but we can only conjecture as to what happened prior to that.

In conclusion, Adam and Eve may therefore be plausibly identified as members of Homo heidelbergensis and as the founding pair at the root of all human species. Challenges to this hypothesis from population genetics fail principally because we cannot rule out on the basis of the genetic divergence exhibited by contemporary humans that our most recent common ancestors, situated more than 500 kya, are the sole genetic progenitors of the entire human race, whether past or present.

Published here with permission, this is the transcript of a Defenders class by Dr. William Lane Craig on May 10, 2020. 


\section{References}

1. This how the term is used by Dennis Venema and others who press argument (1) (Dennis R. Venema and Scot McKnight, Adam and the Genome: Reading Scripture after Genetic Science, Grand Rapids, Mich.: Brazos Press, 2017, pp. 46-48).

2. David Wilcox, "Finding Adam: The Genetics of Human Origins," in Perspectives on an Evolving Creation, ed. Keith B. Miller (Grand Rapids, Mich.: William B. Eerdmans, 2003), p. 252.

3. Venema and McKnight, Adam and the Genome, p. 55.

4. S. Joshua Swamidass, " Heliocentric Certainty Against a Bottleneck of Two?" https://discourse.peacefulscience.org/t/heliocentriccertainty-against-a-bottleneck-of-two/61/4.

5. Personal communication, June 6, 2018.

6. Venema and McKnight, Adam and the Genome, pp. 44, 52, 53, 60.

7. Buggs, "Adam and Eve: a tested hypothesis?"

8. Swamidass, "Heliocentric Certainty."

9. Ibid.

\section{Links}

https://peacefulscience.org/books/genealogical-adam-eve/ https://natureecoevocommunity.nature.com/users/24561-richardbuggs/posts/61200-adam-and-eve-our-ghostly-ancestors

https://peacefulscience.org/books/adam-genome/

https://peacefulscience.org/articles/genealogical-rapprochement/ https://discourse.peacefulscience.org/t/_/61

https://peacefulscience.org/articles/three-stories-on-adam/
10. Ibid.

11. Ola Hössjer and Ann Gauger, "A Single-Couple Human Origin is Possible," BIO-Complexity 1 (2019):1-20. Swamidass, however, thinks that their study has some serious errors.

12. Joshua Swamidass, "Reworking the Science of Adam," http://peacefulscience.org/reworking-adam/.

13. Dennis Venema, “Adam - Once More, with Feeling," (November 24, 2019), https://www.patheos.com/blogs/jesuscreed/2019/11/04/ adam-once-more-with-feeling/. N.B. that this statement confuses necessary and sufficient conditions. Venema should say "only if it happened." Swamidass informs me that in fact the group did not conclude that such a bottleneck would be detectable if it occurred within the last 500,000 years. To say that a bottleneck prior to 500 kya is not detectable does not imply that a bottleneck less than 500 kya is detectable.

14. Venema, "Adam - Once More, With Feeling."

https://discourse.peacefulscience.org/t/_/9283

https://discourse.peacefulscience.org/t/_/10692

https://discourse.peacefulscience.org/t/_/61/4

https://peacefulscience.org/articles/reworking-adam/

https://www.patheos.com/blogs/jesuscreed/2019/11/04/adam-oncemore-with-feeling 